\title{
Réfléchir sur la gouvernance du JAMC
}

$\mathrm{L}$ a question de l'indépendance rédactionnelle des journaux scientifiques a refait surface comme enjeu fondamental de débat à la suite des événements récents survenus au $J A M C^{1-3}$. Le concept n'est pas simple. De quelle façon l'indépendance de la rédaction se rapporte t-elle à la mission du journal? Qui définit la mission? À qui, de quoi et comment le rédacteur en chef doit il rendre compte? Quelle est la meilleure façon de résoudre les conflits entre le rédacteur en chef, l'organisme parrain et l'éditeur?

Suite à ces récents événements, l'AMC a apporté des changements importants à la structure provisoire de gouvernance du $J A M C$ et nommé le très honorable Antonio Lamer, $\mathrm{CP}, \mathrm{CC} \mathrm{CD}$, à la présidence d'un Groupe d'étude de la gouvernance qui doit présenter un rapport intérimaire d'ici la fin mai et un rapport final en juillet $2006^{4,5}$.

En tant que responsables intérimaires du JAMC qui avons à cœur d'assurer la subsistance et la protection du journal, nous ne pouvons insister suffisamment sur notre profonde conviction : l'indépendance rédactionnelle est la condition d'existence absolue de tout journal savant. Sans elle, il est évident que le JAMC ne conservera pas sa place de principal journal médical général critiqué par les pairs au Canada, ni ne la méritera. Voilà qui est très clair. Certaines questions demeurent toutefois sans réponse : que signifie l'indépendance de la rédaction et devrait-on lui imposer des limites? La situation du JAMC a même incité l'Exécutif de l'association mondiale des rédacteurs médicaux (la WAME, World Association of Medical Editors) à demander à son comité de politique rédactionnelle de réexaminer son énoncé actuel ${ }^{6}$ sur l'indépendance de la rédaction pour voir s'il devrait y avoir des limites à cette indépendance.

Nous soulevons ici des enjeux que nous jugeons essentiels aux politiques d'indépendance de la rédaction et aux structures de gouvernance. Ces enjeux nous ont donné du mal lors de la préparation de notre présentation au comité Lamer (www.jamc.ca).

Il existe un lien entre l'indépendance de la rédaction et la mission d'un journal. Donc, comment la mission du JAMC devrait-elle être établie et maintenue avec le temps? Quels sont les rôles respectifs du rédacteur en chef, de l'organisme parrain (l'AMC) et de l'éditeur (s'il est différent de l'organisme parrain) lors de la formulation de la mission? Dans un journal comme le $J A M C$, qui constitue l'un des avantages offerts aux membres de l'association, soit l'AMC, les membres devraient-ils jouer avoir leur mot à dire dans la formulation de la mission du journal? Si tel est le cas, quelle est la meilleure façon de concrétiser leurs opinions, vu le grand éventail de membres de l'AMC (p. ex., citadins, habitants des régions rurales ou éloignées, universi은 taires, intervenants communautaires, chercheurs, cliniciens, éducateurs, administrateurs et protecteurs des patients)?

Lorsque la mission du journal est formulée, qui décide de son interprétation? L'indépendance rédactionnelle s'étend-elle aussi aux décisions et actions de la rédaction qui sont incompatibles avec la mission du journal? En présence de désaccords, quel processus d'arbitrage tranchera et qui doit y avoir recours?
Devrait-on imposer des limites à l'indépendance de la rédaction en ce qui a trait aux points de vue adoptés, au programme de publication retenu, à la partialité et aux intérêts? Les rédacteurs en chef doivent certes bénéficier de l'indépendance, mais nous réalisons qu'ils ont leurs propres perspectives, opinions et intérêts. Le JAMC exige que les auteurs énumèrent les conflits d'intérêt possibles et présentent les partialités et les limites des études, et exige un examen par les pairs rigoureux de tous les rapports de recherche et études afin de cerner les partialités, d'examiner les conflits d'intérêt déclarés et de scruter les limites d'études. Devrait-on imposer les mêmes exigences d'examen par les pairs et de déclaration de conflits d'intérêts à tous les autres éléments de contenu rédactionnel du journal? Devrait on surveiller de près les décisions de la rédaction au sujet du contenu du journal et, le cas échéant, qui devrait surveiller quoi?

L'indépendance rédactionnelle ne devrait pas immuniser contre l'imputabilité. Même lorsqu'il exerce son indépendance, le rédacteur en chef doit quand même être tenu de respecter la mission du journal et d'exercer avec responsabilité son autorité rédactionnelle. Mais à qui le rédacteur doit-il rendre compte? Est il juste de dire que les rédacteurs en chef doivent rendre compte principalement aux lecteurs? Et si oui, aux lecteurs membres de l'Association, aux lecteurs canadiens en général ou aux lecteurs étrangers? Serait-il plutôt préférable que les rédacteurs en chef rendent compte à l'organisme parrain ou au grand public?

Une fois établis la mission du journal et les rapports d'imputabilité, comment peut-on faire l'équilibre entre le double besoin d'indépendance de la rédaction et d'imputabilité? Il faut être extraordinairement sensible à ces problèmes pour choisir la structure de gouvernance.

Les réponses à ces questions non seulement aideront les futurs rédacteurs en chef du JAMC, l'AMC et l'éditeur et rendront le journal plus fort, mais elles aideront aussi les rédacteurs en chef d'autres journaux et leurs organismes parrains. Nous invitons les lecteurs à participer à ce dialogue crucial. - Noni MacDonald, Rédactrice en chef intérim; Bruce Squires, Rédacteur émérite; Jocelyn Downie, Conseillère auprès du Conseil de rédaction intérim; et le Conseil de rédaction intérim : Arnold Aberman, Paul W. Armstrong, Frank Davidoff, Allan Detsky, Judith Hall, Brian Hennen, Jean Rouleau, Claude Roy, Jeff Scott et Donna Stewart

\section{RÉFÉRENCES}

I. Kassirer JP, Davidoff F, O'Hara K, et al. Editorial autonomy of $C M A J$ [éditorial]. JAMC 2006; 174:945-50.

2. Choi S, Flegel $\mathrm{K}$, Kendall $\mathrm{C}$, et al. Un catalyseur de changement [éditorial]. JAMC 2006;174:903.

3. MacDonald N, Squires B. Pourquoi nous avons accepté d'assurer l'intérim [éditorial]. JAMC 2006; I74:1065.

4. Association médicale canadienne. $C M A J$ editorial governance principles Disponible à : www.cmaj.ca/pdfs/governance.pdf (consulté le 24 avril 2006).

5. Collins-Nikai R. CMAJ: moving forward [lettre]. Disponible à : www.cmaj.ca/cgi |eletters?lookup=by_date\&days=2I\# I74/8/1063-a (consulté le 24 avril 2006).

6. World Association of Medical Editors (WAME). Editorial independence. Disponible à : www.wame.org/wamestmt.htm\#independence (consulté le 24 avril 2006). 